\title{
Is Pathologic Complete Response the Surrogate in Primary Gastric Angiosarcoma Undergoing Doxorubicin-Based Neoadjuvant Chemotherapy? A Case Report
}

This article was published in the following Dove Press journal:

International Journal of General Medicine

\author{
Chun-Hui Zheng ${ }^{1,2}$ \\ Yang Wang ${ }^{3}$ \\ Ting-Ting Liu ${ }^{4}$ \\ Xiao-Yan Ding $\left(\mathbb{D}^{5}\right.$ \\ Jian-Jun $\mathrm{Qu}^{2, *}$ \\ Zhi-De Su $\mathbb{I D}^{6, *}$ \\ 'Cheeloo College of Medicine, Shandong \\ University, Jinan, Shandong, People's \\ Republic of China; ${ }^{2}$ Department of \\ Oncology Surgery, Weifang People's \\ Hospital, Weifang, Shandong, People's \\ Republic of China; ${ }^{3}$ Department of \\ General Surgery, Weifang People's \\ Hospital, Weifang, Shandong, People's \\ Republic of China; ${ }^{4}$ Department of Breast \\ Surgery, Tai'an Central Hospital, Tai'an, \\ Shandong, People's Republic of China; \\ ${ }^{5}$ Department of Laboratory Medicine, \\ Weifang Medical University, Weifang, \\ Shandong, People's Republic of China; \\ ${ }^{6}$ Department of Pharmacy, Weifang \\ People's Hospital, Weifang, Shandong, \\ People's Republic of China
}

*These authors contributed equally to this work

Correspondence: Jian-Jun Qu Department of Oncology Surgery, Weifang People's Hospital, I5I Guangwen Street, Weifang, Shandong 26104I, People's Republic of China Email urodoc@l63.com

Zhi-De Su

Department of Pharmacy, Weifang People's Hospital, I5I Guangwen Street, Weifang, Shandong 26I04I, People's Republic of China

Email zchzlwk@I63.com
Introduction: Angiosarcoma is a malignant tumor with low incidence. Especially in the advanced tumors, there is still a lack of knowledge of evidence-based medicine.

Case Presentation: We report a case of a 55-year-old woman with abdominal pain of 2 months of duration, which had increased in severity for 2 weeks prior to the presentation. The diagnosis is primary gastric angiosarcoma. We performed multiple disciplinary team (MDT), and doxorubicin-based neoadjuvant chemotherapy (NAC) was proposed. After two cycles of NAC, a computed tomography (CT) scan showed complete regression compared with the previous scan. An open surgery was done, and surgical specimens were confirmed as a pathological complete response (PCR) by pathological and immunohistochemical examination, but unfortunately, the patient suffered a relapse after the surgery in 3 months. Conclusion: Repeated endoscopic biopsy and biopsy specimen examinations can improve accuracy in diagnosis. It seems that NAC could be a candidate for advanced primary gastric angiosarcomas. But after the rapid relapse, we are wondering whether pathologic complete response is the surrogate in primary gastric angiosarcoma undergoing NAC.

Keywords: gastric angiosarcoma, stomach, neoadjuvant chemotherapy, pathologic complete response

\section{Introduction}

Angiosarcoma is a rare neoplasm type that accounts for less than $2 \%$ of all sarcomas. ${ }^{1}$ It is an infrequent tumor that can develop anywhere in the body. Twothirds of such tumors are cutaneous, mainly found in the head and neck area. ${ }^{1}$ Onefourth arise in soft tissue. In the abdomen, angiosarcomas generally involve the liver and spleen, ${ }^{1}$ but have been rarely reported in the stomach. Among reported cases, only a few were primary gastric angiosarcomas.

Currently, no standardized therapeutic approach is available for these types of sarcomas. Resection for non-metastatic disease, chemotherapy, and radiation are the recommended treatments. ${ }^{1}$ Much of the published data on these rare tumors are based on case series, which causes difficulty in the interpretation of results. Reporting may be selective or incomplete. The site of occurrence is not consistent, and treatment approaches are diverse, even within the same institution, especially with chemotherapy. Currently, chemotherapies used for angiosarcoma include traditional agents, such as 
paclitaxel, docetaxel, and doxorubicin. Angiogenesis inhibitors, drugs that target the growth of endothelial cells, have also been reported. ${ }^{2,3}$ However, these reports mainly describe skin, breast, colon, or mixed tumors due to rare incidence. ${ }^{1,4}$ In this report, we demonstrate the response of a patient with primary gastric angiosarcoma to doxorubicin-based NAC after three cycles. We present the following case in accordance with the CARE reporting checklist. ${ }^{5}$

\section{Case Presentation}

A previously healthy 55-year-old woman was referred to our clinic for abdominal and right shoulder pain. A 40-mm ulcerative lesion within the gastric antrum on the posterior wall of the lower body was identified by esophagogastroduodenoscopy (EGD), the surface of the lesion was irregular, erosive, and reddish (Figure 1). On admission, physical examinations were unremarkable, and the abdominal tumor could not be palpated. Laboratory data were essentially within normal limits, stool occult blood was negative, and the carcinoembryonic antigen level was $5 \mathrm{ng} / \mathrm{dL}$ (normal $<5 \mathrm{ng} / \mathrm{dL}$ ).

Contrast-enhanced abdominal computed tomography (CT) and Positron Emission Tomography-Computed Tomography (PET-CT) showed a mass in the mid-portion of the stomach with marked thickening of the gastric wall (Figures 2 and 3). Due to the uncommon immunohistochemical (IHC) presentation, repeated endoscopic biopsy and biopsy specimen examination were performed. Despite the previous IHC report, even our experienced pathologist was uncomfortable with a gastric angiosarcoma diagnosis

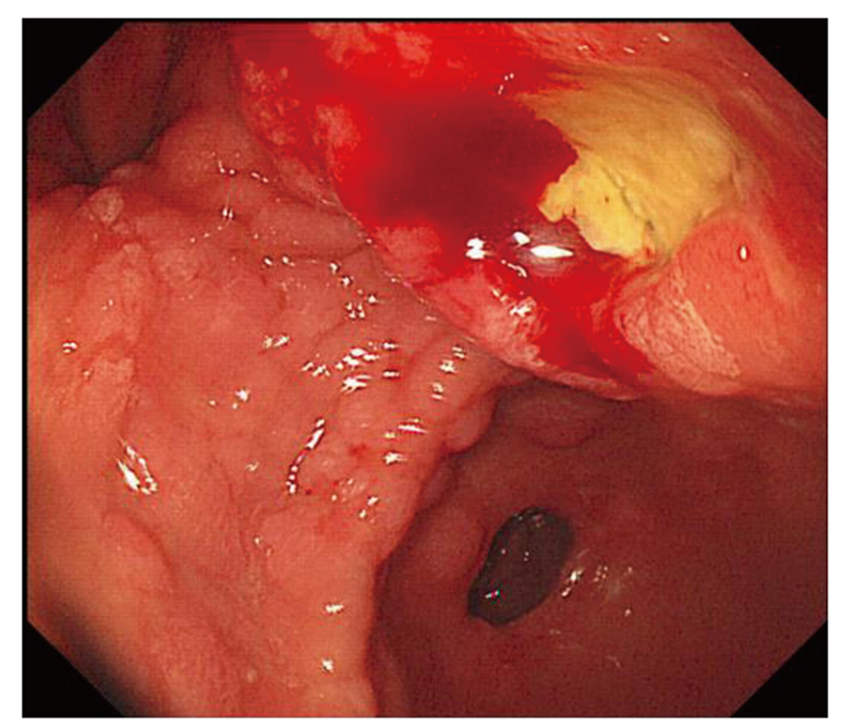

Figure I A tumor measuring $30 \times 40 \mathrm{~mm}$ in diameter was identified in the anterior wall of the gastric corpus.

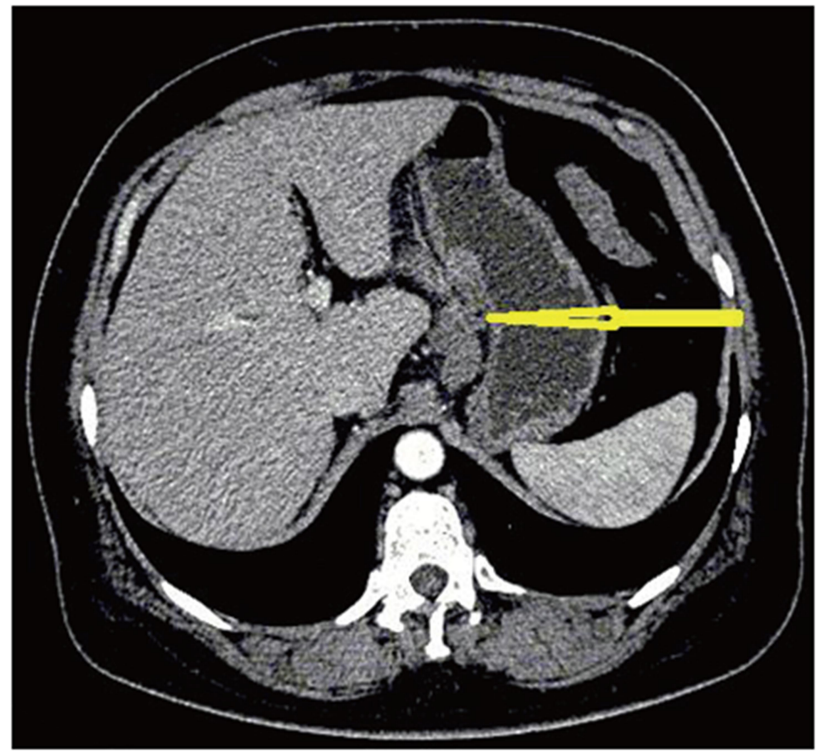

Figure 2 Thickening of the gastric wall (arrowhead), high density of fat around the gastric wall, and the "station 3" enlarged lymph node.

due to its low probability of occurrence. In the immunohistochemical study, the tumor cells were positive for vimentin and $\mathrm{CD} 34$, focally positive for cytokeratin (AE1/AE3), and negative for CD117 (Figure 4A and B). These findings confirmed the diagnosis of mixed epithelioid and moderately differentiated primary gastric angiosarcoma.

No standard guide or treatment experience was available for reference. The patient went to several hospitals without getting a standard answer. A multidisciplinary team (MDT) discussion was conducted for the patient regarding what to do next, whether to do surgery, chemotherapy, or otherwise. Doxorubicin-based NAC was then proposed because our MDT was concerned about the "station 3" lymph node enlargement, and because the rate of short-term survival is uncertain. The patient received chemotherapy $\left(90 \mathrm{mg} / \mathrm{m}^{2}\right.$ doxorubicin on day $1,65 \mathrm{mg} / \mathrm{m}^{2}$ cyclophosphamide on day $1,80 \mathrm{mg} / \mathrm{m}^{2}$ cisplatin on day 1 , repeated every 3 weeks). After two cycles of NAC, the tumor response to chemotherapy was evaluated using an abdominal and pelvic-enhanced CT scan and an EGD, following the Response Evaluation Criteria in Solid Tumors (RECIST) guideline. Complete response was defined by the MDT (Figures 5 and 6). Moreover, clinical symptoms were relieved. The patient underwent an open surgery after the third cycle, and an extended lymph node resection (D2) and gastrectomy were performed. Surgical specimens were assessed to complete the pathological response (Figure 7), the tumor was resected with negative margins and 0/42 lymph nodes being positive for metastatic disease. The regimens of NAC were selected in 


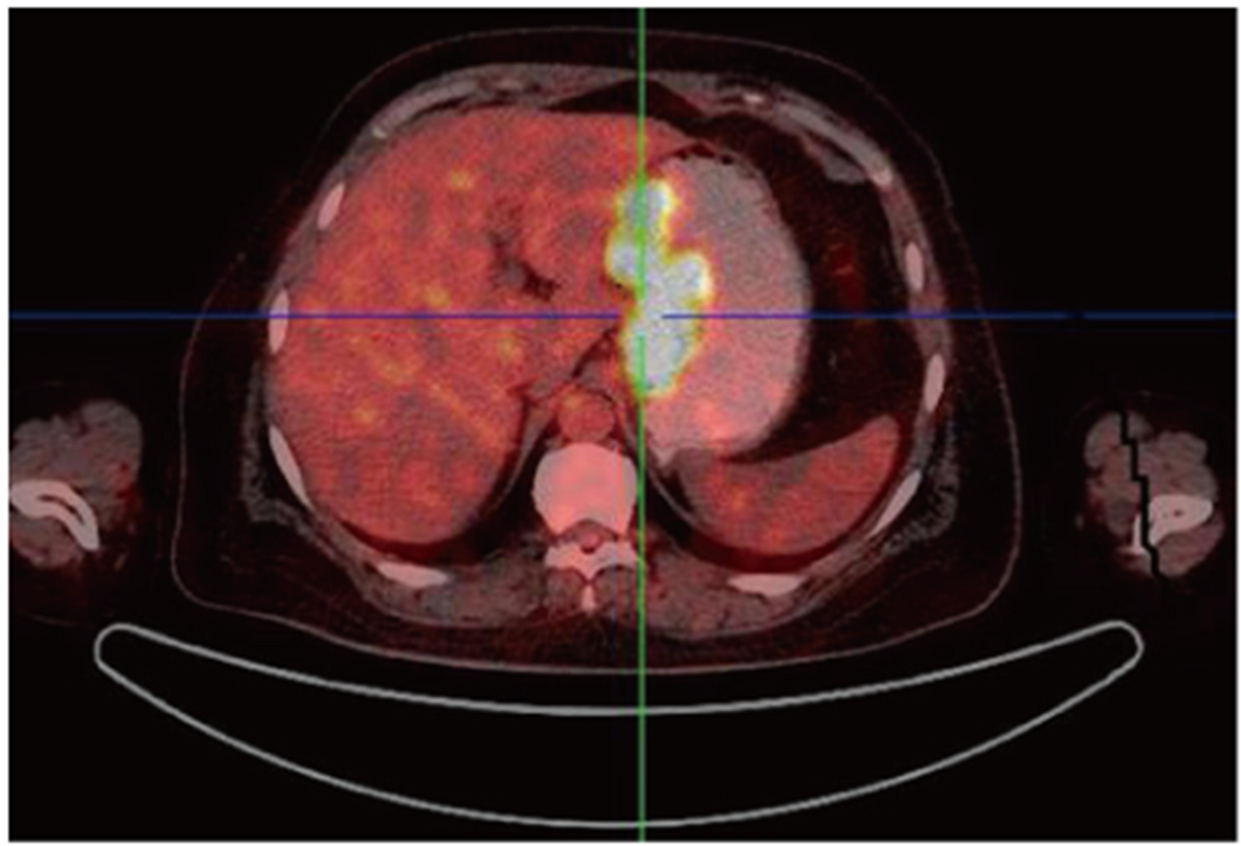

Figure 3 Positron emission tomography/computed tomography revealed uptake by the gastric wall and perigastric lymphadenopathy.

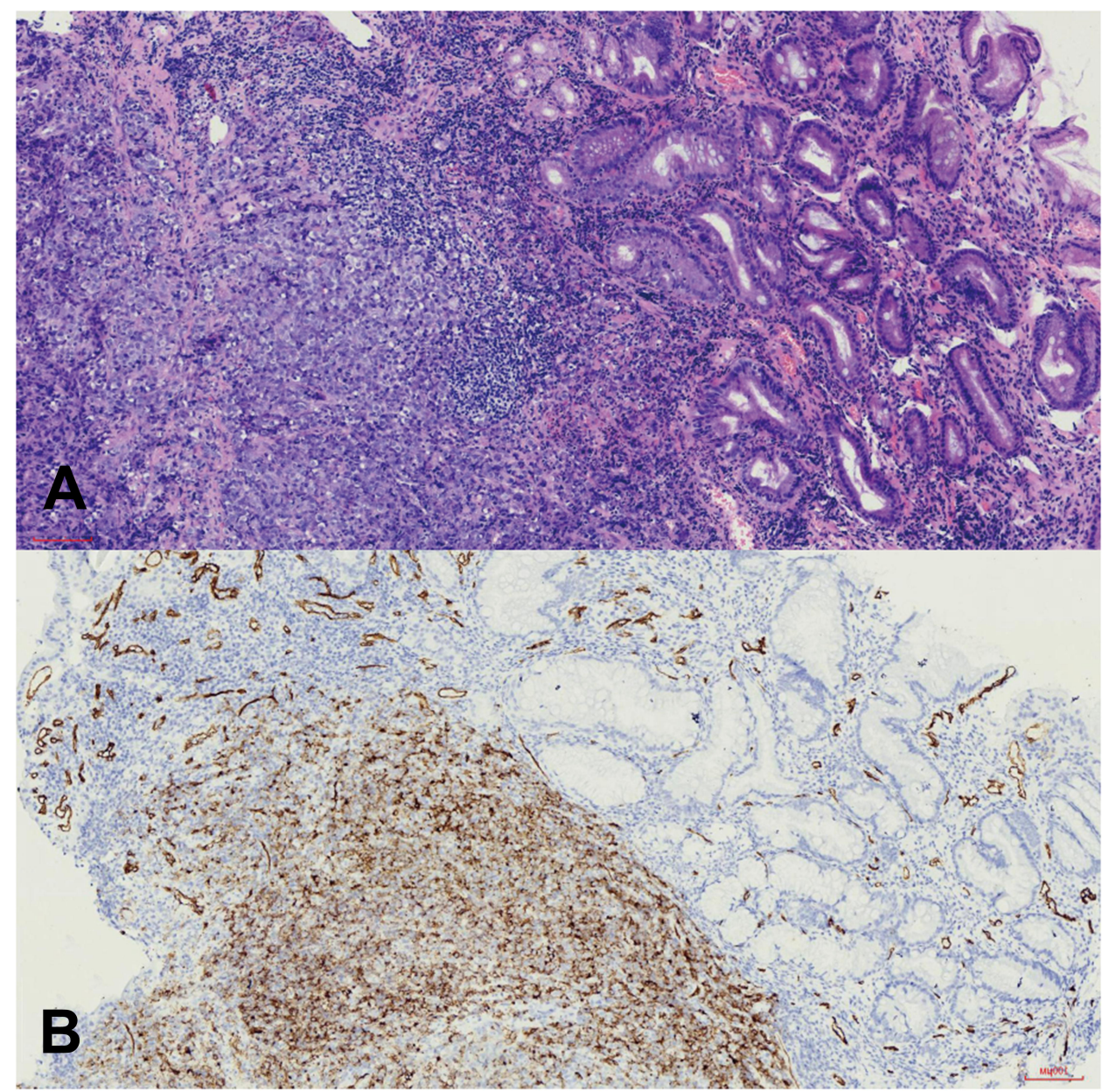

Figure 4 (A) Histopathological findings: Hematoxylin-and-eosin staining of the tumor. (B) On immunohistochemical study, the tumor cells were positive for CD34. 


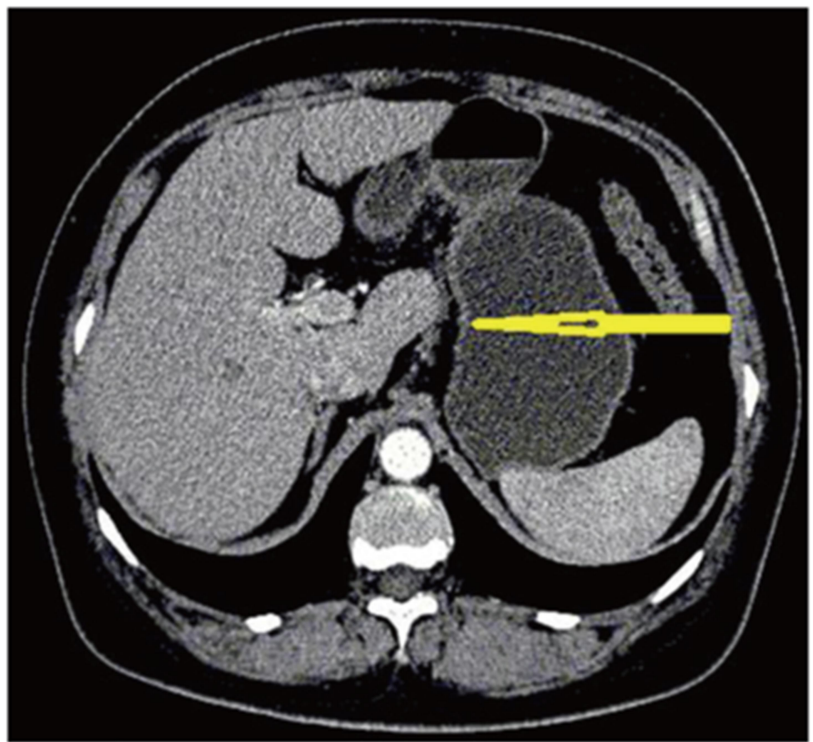

Figure 5 Remarkable shrinkage in tumor size was observed, improvement of gastric wall thickness after chemotherapy and shrinkage of lymphadenopathy after chemotherapy (arrowhead).

adjuvant chemotherapy treatment. During the adjuvant chemotherapy, the disease recurred in the first following reexamination 3 months post-operatively. Contrast-enhanced abdominal CT showed multiple metastases of liver and retroperitoneal region (Figure 8). Then, the patient gave up treatment because of the family poverty. Written informed consent was obtained from the patient for publication of this manuscript and any accompanying images.

\section{Discussion}

To our knowledge, this is the first report of the use of NAC for gastric angiosarcoma and verifies its efficacy. Angiosarcoma involving the stomach is extremely rare reported. Preoperative diagnosis is quite difficult. The clinical presentation of this disease varies. Abdominal pain, abdominal masses, weight loss, and melena are common symptoms. ${ }^{1}$ In this case, the main symptoms were abdominal and right shoulder pain. The radiographic technique has a limited effect on the diagnostic utility due to a lack of specificity. Upper gastrointestinal endoscopy revealed multiple reddish nodules in the stomach and duodenum. Endoscopy can directly detect neoplasia, and it is useful in locations, including stomach, duodenum, and colon. ${ }^{6}$ It needs to be distinguished from adenocarcinoma or gastrointestinal stromal tumor. In our patient's case, most biopsy

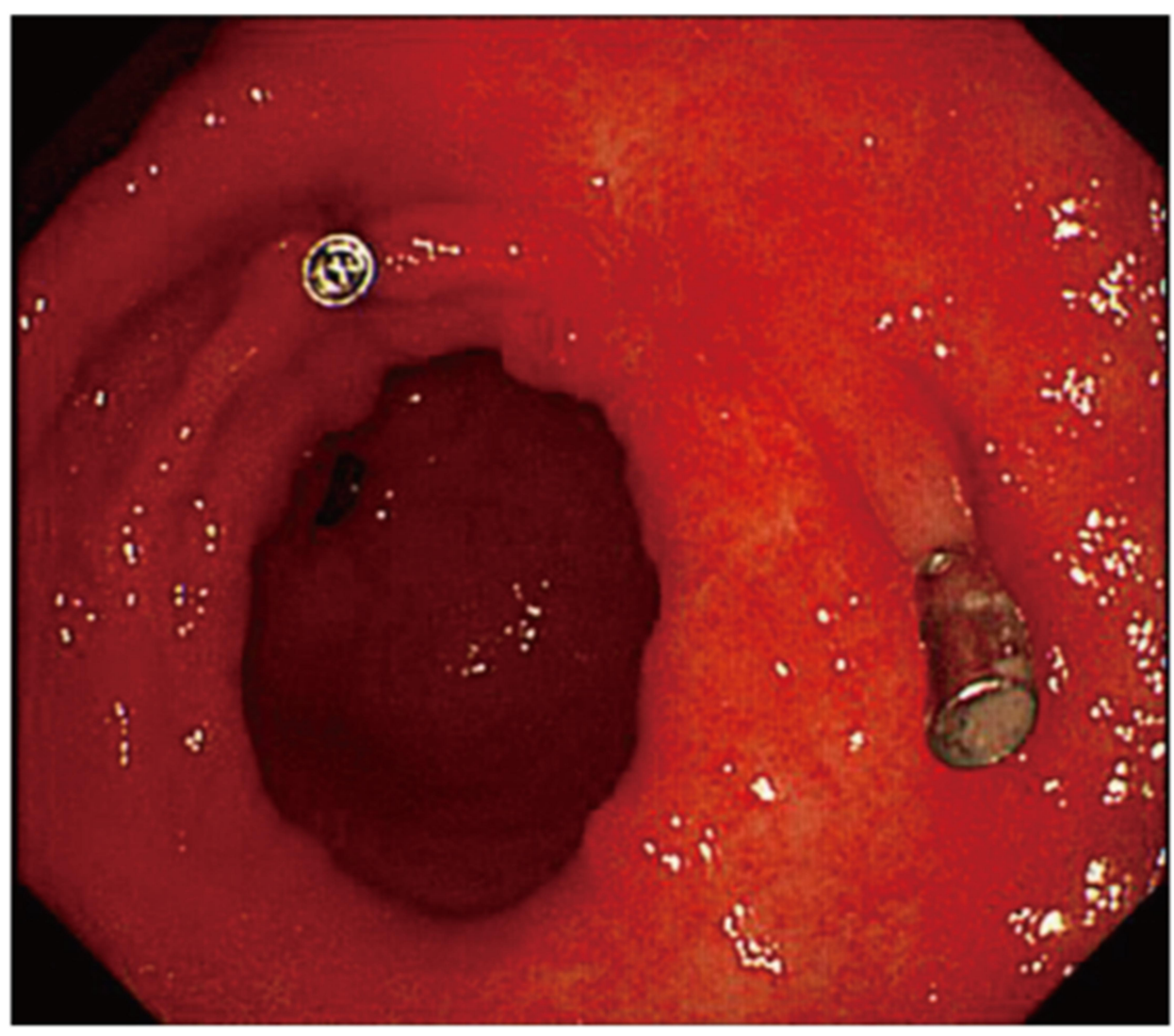

Figure 6 After three cycles of chemotherapy, remarkable shrinkage in tumor size was observed. 


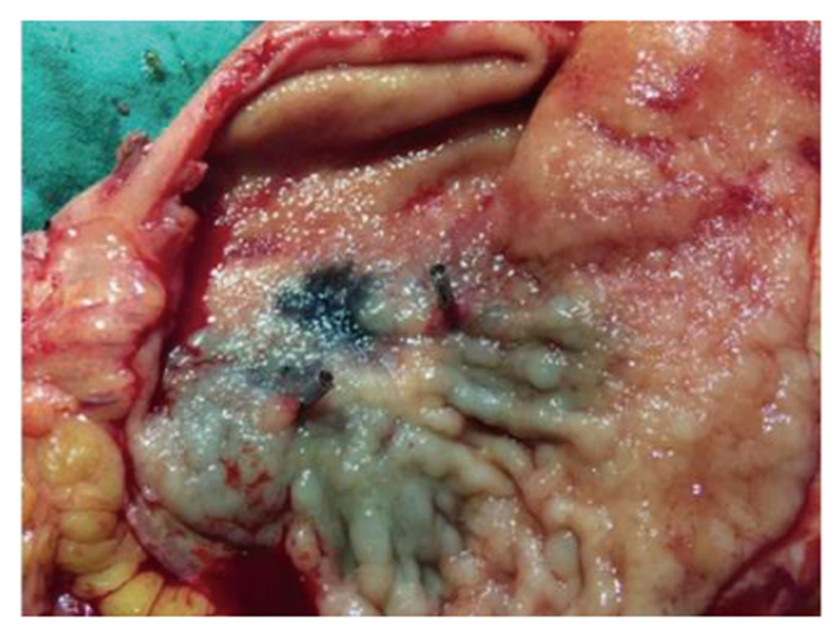

Figure 7 The resected gastric tissue specimen of the angiosarcoma regressed.

specimens showed no evidence of tumor from histological features. In situations like this, it might be helpful to perform endoscopy again because the endoscopic features were unusual. Furthermore, it was useful in this case to examine the biopsy specimen again. In our case, a repeated endoscopic biopsy of the stomach was performed, and pathological and
IHC examinations were done three times. At the worst, in some situations, a definitive diagnosis of angiosarcoma is only possible with exploratory surgery. Emergency surgery is always required when there is uncontrolled bleeding, hemodynamic instability, or perforation. ${ }^{7}$ Therefore, in the literature, only a few cases were diagnosed preoperatively.

Usually, early detection and complete surgical resection followed by chemotherapy may be the most effective treatment regimen in some common malignancies. A previous literature on gastric angiosarcoma was reviewed to find any effective treatment, but we found that the efficacy of NAC for angiosarcoma was unclear, and guidelines for NAC and adjuvant therapy were not well described. ${ }^{8}$ Clinical studies on gastric angiosarcomas are inconclusive due to their rarity. Their results are also diverse, and generally showed poor prognosis. ${ }^{4,9}$

Reports of cases involving other sites of angiosarcoma were reviewed. Early reports showed poor experience in management with adjuvant chemotherapy or combinations of chemotherapy and radiation. ${ }^{10}$ Tse-Hua Lo's report ${ }^{11}$ on the effect of chemotherapy on angiosarcoma was disappointing, but this

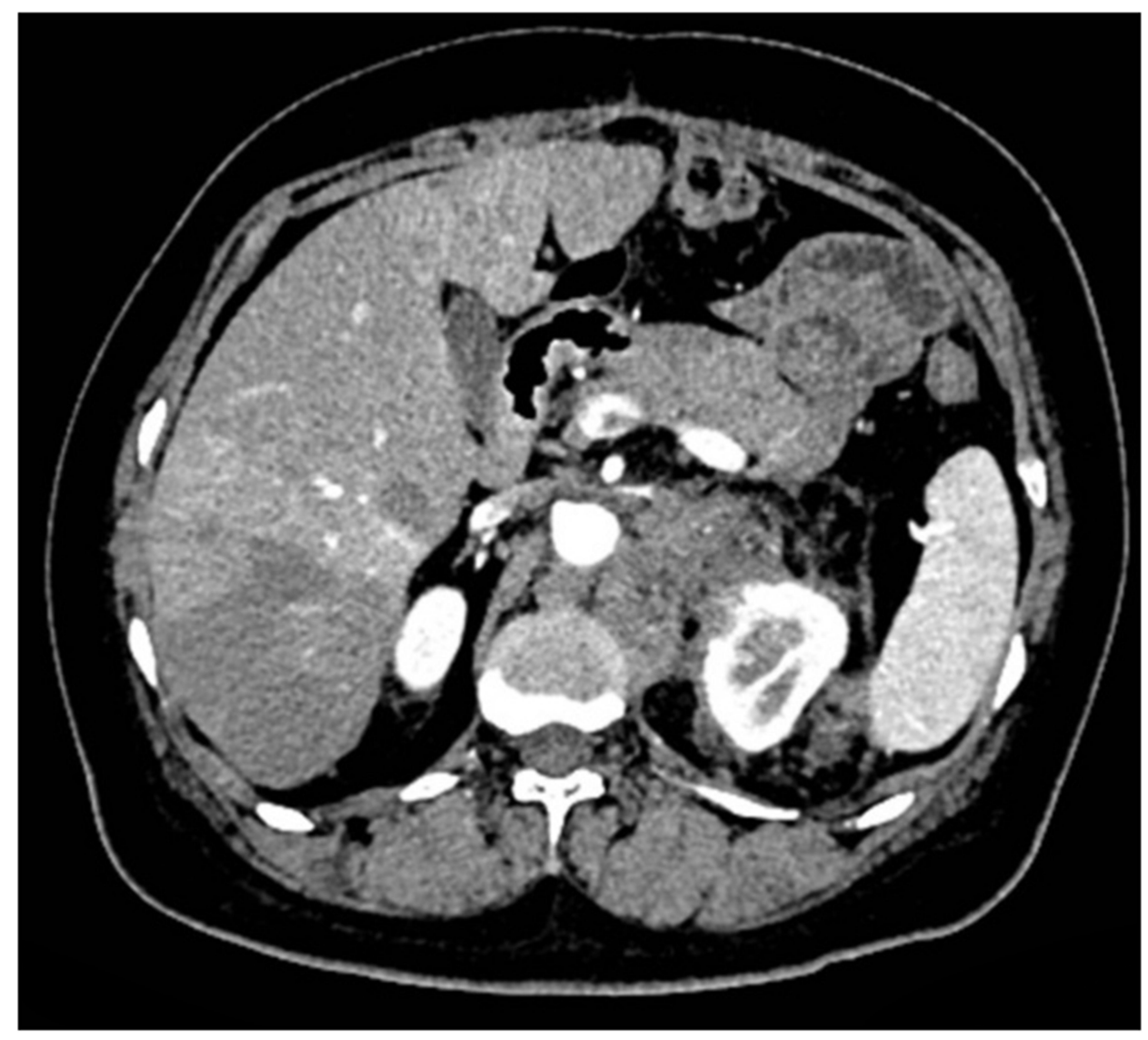

Figure 8 Multiple metastases of liver and retroperitoneal region after the surgery. 
patient was still alive without evidence of recurrence or metastasis at a three-year follow-up appointment. Thus, he concluded that the role of adjuvant treatment for colorectal angiosarcomas was unclear, and curative surgical excision was still the best choice of treatment, which was due to the remarkable clinical heterogeneity of angiosarcoma according to primary locations. ${ }^{12}$ Meanwhile, numerous case reports suggest the potential of chemotherapy for metastatic or advanced angiosarcoma. ${ }^{8,10}$ By analogy with other histological subtypes of soft tissue sarcoma, doxorubicin or taxanebased $^{8,10,12}$ regimens remain the recommended first-line treatments of choice. This has been confirmed by EORTC clinical trials. ${ }^{10}$ The response rate, progression-free survival, and overall survival are about 25\%, 4.9 months, and 9.9 months, respectively.

Remarkably, doxorubicin-based chemotherapy regimens, which are commonly used for other sarcoma subtypes, ${ }^{10}$ have also been used for angiosarcomas. Skubitz and Haddad ${ }^{13}$ reported their experience with 13 patients initially treated with either paclitaxel or pegylated liposomal doxorubicin. Five out of eight patients who were treated with paclitaxel had either partial or complete responses, and three of six patients who were treated with pegylated-liposomal doxorubicin had partial responses. Abraham reported that advanced patients were generally treated with doxorubicin-based regimens or vinorelbine, and $64 \%$ of the patients had at least a partial response to at least one regimen. ${ }^{14}$ Our case was discussed by an MDT. Considering the "station 3" lymph node enlargement shown on PET-CT and the uncertainty about the short-term survival as based on the literature, surgical therapy was not accepted by the patient and her relatives. Doxorubicin-based chemotherapy was then proposed by the MDT. However, there has been no Phase II trial in gastric angiosarcoma. Fortunately, the effect of NAC was positive from the imaging point of view.

We have here described a gastric angiosarcoma that successfully responded to doxorubicin-based NAC. But after a long and painful course of chemotherapy and surgery, the patient suffered a relapse. Satisfactory NAC results did not lead to satisfactory prognosis after the surgery.

\section{Conclusions}

Primary gastric angiosarcoma is extremely rare, and preoperative diagnosis is quite difficult. For advanced patients, exposure to chemotherapy at the earliest time may allow them to be successfully converted to a resectable tumor by NAC. Although no phase II trials have been performed based on the available evidence, treatment with a doxorubicin-based regimen may be acceptable. Randomized trials make it challenging to perform treatment in such a rare disease, but ideally, a prospective randomized trial to compare NAC with immediate surgery in the patient should be performed in order to provide an answer to this question.

\section{Ethics Approval and Consent to Participate}

Based on the regulation of the department of research of Weifang People's Hospital, institutional review board approval is not required to publish the case details. Written informed consent has been provided by the patient and her son to have the case details and any accompanying images published.

\section{Author Contributions}

All authors made a significant contribution to the work reported, whether that is in the conception, study design, execution, acquisition of data, analysis and interpretation, or in all these areas; took part in drafting, revising or critically reviewing the article; gave final approval of the version to be published; have agreed on the journal to which the article has been submitted; and agree to be accountable for all aspects of the work.

\section{Funding}

This work was supported by the Medical and Health Science Technology Development Program in Shandong Province (2019WS254) and Science Technology Development Program in Weifang City (2019YX002).

\section{Disclosure}

Zhi-De Su and Chun-Hui Zheng report grants from the Medical and Health Science Technology Development Program in Shandong Province, during the conduct of the study. The authors have no other potential conflicts of interest to declare.

\section{References}

1. Young RJ, Brown NJ, Reed MW, Hughes D, Woll PJ. Angiosarcoma. Lancet Oncol. 2010;11(10):983-991. doi:10.1016/S1470-2045(10) 70023-1

2. Penel N, Bui BN, Bay JO, et al. Phase II trial of weekly paclitaxel for unresectable angiosarcoma: the ANGIOTAX Study. J Clin Oncol. 2008;26(32):5269-5274. doi:10.1200/JCO.2008.17.3146

3. Chen TW, Burns J, Jones RL, Huang PH. Optimal clinical management and the molecular biology of angiosarcomas. Cancers (Basel). 2020;12(11):E3321. doi:10.3390/cancers12113321 
4. Constantinidou A, Sauve N, Stacchiotti S, et al. Evaluation of the use and efficacy of (neo)adjuvant chemotherapy in angiosarcoma: a multicentre study. ESMO Open. 2020;5(4):e000787. doi:10.1136/ esmoopen-2020-000787

5. Riley DS, Barber MS, Kienle GS, et al. CARE guidelines for case reports: explanation and elaboration document. J Clin Epidemiol. 2017;89:218-235. doi:10.1016/j.jclinepi.2017.04.026

6. Allison KH, Yoder BJ, Bronner MP, Goldblum JR, Rubin BP. Angiosarcoma involving the gastrointestinal tract: a series of primary and metastatic cases. Am J Surg Pathol. 2004;28(3):298-307. doi:10.1097/00000478-200403000-00002

7. Navarro-Chagoya D, Figueroa-Ruiz M, López-Gómez J, et al. Obscure gastrointestinal bleeding due to multifocal intestinal angiosarcoma. Int J Surg Case Rep. 2015;10:169-172. doi:10.1016/ j.ijscr.2015.03.049

8. Casali PG, Abecassis N, Aro HT, et al. Soft tissue and visceral sarcomas: ESMO-EURACAN clinical practice guidelines for diagnosis, treatment and follow-up. Ann Oncol. 2018;29(Suppl4):iv268iv269. doi:10.1093/annonc/mdy321

9. Mehta CR, Liu L, Theuer C. An adaptive population enrichment Phase III trial of TRC105 and pazopanib versus pazopanib alone in patients with advanced angiosarcoma (TAPPAS trial). Ann Oncol. 2019;30(1):103-108. doi:10.1093/annonc/mdy464
10. Young RJ, Natukunda A, Litière S, Woll PJ, Wardelmann E, van der Graaf WT. First-line anthracycline-based chemotherapy for angiosarcoma and other soft tissue sarcoma subtypes: pooled analysis of eleven European Organisation for Research and Treatment of Cancer Soft Tissue and Bone Sarcoma Group trials. Eur J Cancer. 2014;50(18):3178-3186. doi:10.1016/j.ejca.2014.10.004

11. Lo TH, Tsai MS, Chen TA. Angiosarcoma of sigmoid colon with intraperitoneal bleeding: case report and literature review. Ann R Coll Surg Engl. 2011;93(6):e91-93. doi:10.1308/147870811X591017

12. Fujisawa Y, Yoshino K, Kadono T, Miyagawa T, Nakamura Y, Fujimoto M. Chemoradiotherapy with taxane is superior to conventional surgery and radiotherapy in the management of cutaneous angiosarcoma: a multicentre, retrospective study. $\mathrm{Br} J$ Dermatol. 2014;171(6):1493-1500. doi:10.1111/bjd.13110

13. Skubitz KM, Haddad PA. Paclitaxel and pegylated-liposomal doxorubicin are both active in angiosarcoma. Cancer. 2005;104 (2):361-366. doi:10.1002/cncr.21140

14. Abraham JA, Hornicek FJ, Kaufman AM, et al. Treatment and outcome of 82 patients with angiosarcoma. Ann Surg Oncol. 2007;14 (6):1953-1967. doi:10.1245/s10434-006-9335-y
International Journal of General Medicine

\section{Publish your work in this journal}

The International Journal of General Medicine is an international, peer-reviewed open-access journal that focuses on general and internal medicine, pathogenesis, epidemiology, diagnosis, monitoring and treatment protocols. The journal is characterized by the rapid reporting of reviews, original research and clinical studies across all disease areas. The manuscript management system is completely online and includes a very quick and fair peer-review system, which is all easy to use. Visit http://www.dovepress.com/ testimonials.php to read real quotes from published authors. 